\title{
СЛАБОЕ НЕРАВЕНСТВО МАРКОВА ДЛЯ АЛГЕБРАИЧЕСКИХ МНОГОЧЛЕНОВ НА ОТРЕЗКЕ
}

\section{Н. С. Паюченко}

\begin{abstract}
Для вещественного алгебраического многочлена $P_{n}$ степени $n$ рассмотрим отношение $M_{n}\left(P_{n}\right)$ меры множества точек отрезка $[-1,1]$, в которых модуль производной превосходит $n^{2}$, к мере множества точек, в которых модуль многочлена превосходит 1. Изучается точная верхняя грань $M_{n}=\sup M_{n}\left(P_{n}\right)$ по множеству многочленов $P_{n}$ с равномерной нормой на $[-1,1]$, большей 1 . Известно, что величина $M_{n}$ является супремумом точных констант в неравенстве Маркова по классу интегральных функционалов, порожденных неубывающей неотрицательной функцией. Результатом работы являются следующие оценки: $1+3 /\left(n^{2}-1\right) \leq M_{n} \leq 6 n+1, n \geq 2$.
\end{abstract}

Ключевые слова: неравенство Маркова, алгебраические многочлены, мера Лебега, неравенства слабого типа.

N. S. Payuchenko. Markov's weak inequality for algebraic polynomials on a closed interval.

For a real algebraic polynomial $P_{n}$ of degree $n$, we consider the ratio $M_{n}\left(P_{n}\right)$ of the measure of the set of points from $[-1,1]$ where the absolute value of the derivative exceeds $n^{2}$ to the measure of the set of points where the absolute value of the polynomial exceeds 1 . We study the supremum $M_{n}=\sup M_{n}\left(P_{n}\right)$ over the set of polynomials $P_{n}$ whose uniform norm on $[-1,1]$ is greater than 1 . It is known that $M_{n}$ is the supremum of the exact constants in Markov's inequality in the class of integral functionals generated by a nondecreasing nonnegative function. In this paper we prove the estimates $1+3 /\left(n^{2}-1\right) \leq M_{n} \leq 6 n+1$ for $n \geq 2$.

Keywords: Markov's inequality, algebraic polynomials, Lebesgue measure, weak-type inequalities.

MSC: 26D10

DOI: $10.21538 / 0134-4889-2019-25-2-160-166$

\section{1. Введение}

Пусть $\mathcal{P}_{n}$ есть множество вещественных алгебраических многочленов $P_{n}$ степени не выше $n$. Для равномерной нормы $\left\|P_{n}\right\|=\max _{[-1,1]}\left|P_{n}(x)\right|$ на $\mathcal{P}_{n}$ хорошо известно неравенство В. А. Маркова [11, Theorem 5.1.8]

$$
\left\|P_{n}^{\prime}\right\| \leq n^{2}\left\|P_{n}\right\|, \quad P_{n} \in \mathcal{P}_{n} .
$$

Оно обращается в равенство на многочленах Чебышева первого рода

$$
T_{n}(x)=\cos (n \arccos x), \quad x \in[-1,1] .
$$

Неравенство (1.1) обобщалось на другие нормы, прежде всего на нормы $L^{q}[-1,1]$ :

$$
\left(\int_{-1}^{1}\left|P_{n}^{\prime}(x)\right|^{q} d x\right)^{1 / q} \leq M_{n}^{q}\left(\int_{-1}^{1}\left|P_{n}(x)\right|^{q} d x\right)^{1 / q}, \quad q>0 .
$$

\footnotetext{
${ }^{1}$ Работа выполнена при поддержке Программы повышения конкурентоспособности УрФУ (Постановление Правительства РФ № 211 от 16 марта 2013 г., соглашение № 02.А03.21.0006 от 27 августа 2013 г.).
} 
Относительно (1.3) доказано, что $M_{n}^{q}$ растет как $n^{2}$ при $n \rightarrow \infty$ и фиксированном $q$. Более подробно историю исследования задачи и конкретные оценки $M_{n}^{q}$ можно посмотреть в работах [1-4].

Обратимся к более общей задаче. Пусть $\Lambda$ - линейный оператор на $\mathcal{P}_{n}, \Phi-$ класс неотрицательных, неубывающих на $[0, \infty)$ функций. Рассмотрим неравенство с точной константой $M_{n}(\Lambda, \phi)$

$$
\int_{-1}^{1} \phi\left(\left|\Lambda P_{n}(x)\right|\right) d x \leq M_{n}(\Lambda, \phi) \int_{-1}^{1} \phi\left(\left|P_{n}(x)\right|\right) d x .
$$

Для функции $\phi(u)=u^{q}$ и оператора $\Lambda P_{n}=P_{n}^{\prime} / n^{2}$ неравенство (1.4) сводится к (1.3), при этом $M_{n}(\Lambda, \phi)^{1 / q} n^{2}=M_{n}^{q}$.

Известно (см., например, [5, Lemma 4.1]), что точная верхняя грань величины $M_{n}(\Lambda, \phi)$ по всем $\phi \in \Phi$ достигается на функции $\phi^{*}(x)=1, x \geq 1, \phi^{*}(x)=0, x<1$. Таким образом, при $\phi=\phi^{*}$ неравенство (1.4) принимает вид

$$
\mu\left(\Lambda P_{n}\right) \leq M_{n}\left(\Lambda, \phi^{*}\right) \mu\left(P_{n}\right), \quad P_{n} \in \mathcal{P}_{n},
$$

где $\mu\left(P_{n}\right)=\operatorname{mes}\left\{x \in[-1,1]:\left|P_{n}(x)\right| \geq 1\right\}-$ мера множества точек $x \in[-1,1]$, в которых $\left|P_{n}(x)\right| \geq 1$.

В настоящей работе изучается точная константа в неравенстве (1.5) для оператора $\Lambda P_{n}=$ $P_{n}^{\prime} / n^{2}$, т. е. величина

$$
M_{n}=\sup _{P_{n} \in \mathcal{P}_{n},\left\|P_{n}\right\|>1} M_{n}\left(P_{n}\right), \text { где } \quad M_{n}\left(P_{n}\right)=\frac{\mu\left(P_{n}^{\prime} / n^{2}\right)}{\mu\left(P_{n}\right)} .
$$

Для оператора $\Lambda P_{n}=P_{n}^{(n)} / n$ ! задача (1.5) сводится к нахождению многочлена с фиксированным старшим коэффициентом, наименее уклоняющегося от нуля по мере

$$
M_{n}^{*}(\Lambda)=\frac{2}{\inf _{\left\|P_{n}\right\|>1} \mu\left(P_{n}\right)} .
$$

Эта задача решена В. В. Арестовым в работе [5]:

$$
\inf _{P_{n-1} \in \mathcal{P}_{n-1}} \mu\left(P_{n}^{y}\right)=2\left(1-y^{-1 / n}\right), \quad P_{n}^{y}(x)=y 2^{n-1} x^{n}+P_{n-1}, \quad|y|>1 .
$$

Равенство достигается на многочлене $T_{n}\left(y^{1 / n} x\right)$.

Аналогичную (1.6) проблему на множестве $\mathcal{T}_{n}$ вещественных тригонометрических полиномов $t_{n}$ порядка $n$ исследовали А. Г. Бабенко [6] и Е. Д. Лившиц [7]. А. Г. Бабенко в [6] рассматривал оператор $\Lambda t_{n}=t_{n}^{\prime} / n$ и получил оценки

$$
\frac{2}{\pi} \ln (2 n+1) \leq \sup _{t_{n} \in \mathcal{T}_{n}} C_{n}\left(t_{n}\right) \leq 2 n, \quad C_{n}\left(t_{n}\right)=\frac{\operatorname{mes}\left\{x \in[0,2 \pi]:\left|t_{n}^{\prime}(x)\right| / n \geq 1\right\}}{\operatorname{mes}\left\{x \in[0,2 \pi]:\left|t_{n}(x)\right| \geq 1\right\}} .
$$

Оценка сверху была уточнена Е. Д. Лившицем [7] на классе $T_{n}^{c}$ полиномов, модуль которых ограничен числом $c>1$ : $\sup _{t_{n} \in T_{n}^{c}} C_{n}\left(t_{n}\right) \leq c_{0}(\ln n+1)$, где $c_{0}$ не зависит от $n$.

\section{2. Оценки величины $M_{n}\left(P_{n}\right)$}

Рассмотрим отдельно многочлены 1 степени. Этот случай является особенным, поскольку для многочленов $a x$ при $a \rightarrow 1+$ имеем $M_{1}(a x) \rightarrow \infty$. В связи с этим изменим постановку 
задачи при $n=1$. Для $d>1$ на множестве многочленов $P_{1}(x)=a x+b$ рассмотрим оператор $\Lambda P_{1}=P_{1}^{\prime} / d=a / d$. Искомая величина есть

$$
M_{1, d}=\sup _{\left\|P_{1}\right\|>1} \frac{\mu(a / d)}{\mu\left(P_{1}\right)}=\sup _{|a|>d} \frac{2}{\mu\left(P_{1}\right)} .
$$

Оценим $\mu\left(P_{1}\right)$. Очевидно, достаточно рассмотреть только $a>d, b \geq 0$ и считать, что $P_{1}(-1) \leq-1$. При этих условиях $\mu\left(P_{1}\right)=1-(1-b) / a+(-1-b) / a+1=2(1-1 / a)$ и

$$
M_{1, d}=\sup _{a>d} \frac{1}{1-1 / a}=\frac{d}{d-1} .
$$

В дальнейших рассуждениях мы несколько раз будем применять неравенство Бернштейна [11, разд. 7]

$$
\left|P_{n}^{\prime}(x)\right| \leq \frac{n}{\sqrt{1-x^{2}}}\left\|P_{n}\right\|, \quad|x|<1, \quad P_{n} \in \mathcal{P}_{n},
$$

и вытекающую из неравенства Маркова (1.1) простую оценку

$$
\mu\left(P_{n}\right) \geq \frac{\left\|P_{n}\right\|-1}{\left\|P_{n}^{\prime}\right\|} \geq \frac{\left\|P_{n}\right\|-1}{n^{2}\left\|P_{n}\right\|} .
$$

Теорема 1. Для $n \geq 2$ справедливы оченки

$$
M_{n}\left(P_{n}\right) \leq 8 \quad \text { при } 1<\left\|P_{n}\right\| \leq 1.5, \quad M_{n}\left(P_{n}\right) \leq \frac{2 c^{3}}{c-1} \quad \text { nрu } \quad 1.5 \leq\left\|P_{n}\right\| \leq c .
$$

Д о к а з а т е л ь с т в о. Обратимся к задаче о максимуме производной многочлена степени $n$ в фиксированной точке $x \in[-1,1]$. Согласно [8, следствие 2] (см. также [9, теорема 1]) на отрезке $[-1,1]$ имеется $n+1$ так называемых чебышевских сегментов $\left[\alpha_{k}, \beta_{k}\right], k=0, \ldots, n$, на которых

$$
\max _{P_{n} \in \mathcal{P}_{n},\left\|P_{n}\right\| \leq M}\left|P_{n}^{\prime}(x)\right|=M T_{n}^{\prime}(x), \quad x \in\left[\alpha_{k}, \beta_{k}\right] .
$$

При этом $\alpha_{0}=-1$, числа $\alpha_{1}, \ldots, \alpha_{n}$ являются корнями уравнения

$$
\frac{d}{d x} \frac{R_{n+1}(x)}{x+1}=0
$$

$\beta_{n}=1$, числа $\beta_{0}, \ldots, \beta_{n-1}$ являются корнями уравнения $\frac{d}{d x} \frac{R_{n+1}(x)}{x-1}=0, R_{n+1}$ - резольвента $T_{n}$. Резольвента имеет вид (см., например, [10, с. 50])

$$
R_{n+1}(x)=\frac{1}{n 2^{n-1}}\left(x^{2}-1\right) T_{n}^{\prime}(x) .
$$

Нас будут интересовать первый и последний чебышевский сегменты $\left[\alpha_{0}, \beta_{0}\right]$ и $\left[\alpha_{n}, \beta_{n}\right]$. С помощью замены $Q_{n}(x)=P_{n}(-x)$ нетрудно убедиться, что $\beta_{0}=-\alpha_{n}$. Величина $\alpha_{n}-$ самое большое решение уравнения (2.4). Оценим $\alpha_{n}$ сверху. Используя представление (2.5), запишем (2.4) в виде

$$
T_{n}^{\prime}(x)-(1-x) T_{n}^{\prime \prime}(x)=0 .
$$

Подставив в (2.6) выражение (1.2), приходим к соотношениям

$$
\begin{gathered}
\frac{n \sin (n \arccos x)}{\sqrt{1-x^{2}}}-(1-x)\left(-\frac{n^{2} \cos (n \arccos x)}{1-x^{2}}+\frac{n x \sin (n \arccos x)}{\left(1-x^{2}\right)^{3 / 2}}\right)=0, \\
n \cos (n \arccos x)+\frac{\sin (n \arccos x)}{\sqrt{1-x^{2}}}=0
\end{gathered}
$$




$$
n^{2} T_{n}(x)+T_{n}^{\prime}(x)=0 .
$$

Очевидно, что все корни уравнения (2.7) меньше наибольшего нуля $T_{n}(x)$, поэтому $\alpha_{n}<$ $\cos (\pi /(2 n))$. Таким образом,

$$
\left|P_{n}^{\prime}(x)\right| \leq\left\|P_{n}\right\|\left|T_{n}^{\prime}(x)\right|, \quad|x| \in[\cos (\pi /(2 n)), 1] .
$$

В силу неравенства Бернштейна (2.1), если

$$
\frac{n}{\sqrt{1-x^{2}}}\left\|P_{n}\right\|<n^{2} \quad \text { или эквивалентно } \quad|x|<\sqrt{1-\left\|P_{n}\right\|^{2} / n^{2}},
$$

то заведомо $\left|P_{n}^{\prime}(x)\right|<n^{2}$. Поэтому при выполнении условия

$$
\sqrt{1-\left\|P_{n}\right\|^{2} / n^{2}} \geq \cos (\pi /(2 n)) \quad \text { или, то же самое }\left\|P_{n}\right\| \leq n \sqrt{1-\cos ^{2}(\pi /(2 n))},
$$

модуль $P_{n}^{\prime}$ может превосходить $n^{2}$ на отрезке $[-1,1]$ только в точках промежутков $[-1,-\cos (\pi /(2 n))]$ и $[\cos (\pi /(2 n)), 1]$. Отсюда и из $(2.8)$ следует, что при условии $(2.10)$

$$
\mu\left(P_{n}^{\prime} / n^{2}\right) \leq \mu\left(\left\|P_{n}\right\| T_{n}^{\prime} / n^{2}\right) .
$$

Видно, что $n \sqrt{1-\cos ^{2}(\pi /(2 n))}=n \sin (\pi /(2 n))$. Функция $u(t)=t \sin (\pi /(2 t))$ при $t \geq 1$ имеет положительную производную и как следствие возрастает, поэтому $1.5=3 \sin (\pi / 6) \leq$ $t \sin (\pi /(2 t)), t \geq 3$. Отсюда следует, что если

$$
1<\left\|P_{n}\right\|<1.5, \quad n \geq 3
$$

то выполняется (2.10) и как следствие (2.11).

Оценим сверху $\mu\left(\left\|P_{n}\right\| T_{n}^{\prime} / n^{2}\right)$ при условии (2.12). Так как $T_{n}^{\prime}$ возрастает при $x>\cos (\pi /(2 n))$, достаточно найти точку $x_{0} \in[\cos (\pi /(2 n)), 1]$, в которой $\left\|P_{n}\right\| T_{n}^{\prime}\left(x_{0}\right)=n^{2}$ :

$$
\left\|P_{n}\right\| \frac{n \sin \left(n \arccos x_{0}\right)}{\sqrt{1-x_{0}^{2}}} \leq n^{2} .
$$

Положим $x_{0}=\cos t_{0}$, тогда

$$
\left\|P_{n}\right\| \sin \left(n t_{0}\right) \leq n \sin t_{0}, \quad n \geq 3
$$

Будем искать $t_{0}$ в виде $t_{0}=\frac{1}{n} \sqrt{\tau \frac{\left\|P_{n}\right\|-1}{\left\|P_{n}\right\|}}$.

Во-первых, найдем, при каких значениях $\tau>0$ ряд Тейлора $\sin \left(n t_{0}\right)$ представляет собой ряд Лейбница. Тогда разложение $\sin t_{0}$, очевидно, также является рядом Лейбница

$$
\frac{\left(n t_{0}\right)^{k+2}}{(k+2) !}<\frac{\left(n t_{0}\right)^{k}}{k !}, \quad \tau \frac{\left\|P_{n}\right\|-1}{\left\|P_{n}\right\|}<(k+1)(k+2), \quad k \in \mathbb{N} .
$$

Подставляя наибольшее значение $\left\|P_{n}\right\|=1.5$ и наименьшее $k=1$, получаем $0<\tau \leq 18$. В силу предыдущих рассуждений справедливы оценки

$$
\left\|P_{n}\right\| \sin \left(n t_{0}\right) \leq\left\|P_{n}\right\|\left(n t_{0}-\left(n t_{0}\right)^{3} / 3 !+\left(n t_{0}\right)^{5} / 5 !\right), \quad n\left(t_{0}-t_{0}^{3} / 3 !\right) \leq n \sin t_{0} .
$$

Таким образом, неравенство (2.13) будет выполнено, если выполняется (2.12) и

$$
\left\|P_{n}\right\|\left(n t_{0}-\left(n t_{0}\right)^{3} / 3 !+\left(n t_{0}\right)^{5} / 5 !\right) \leq n t_{0}-n t_{0}^{3} / 3 ! .
$$

Умножим (2.14) на $5 ! /\left(n t_{0}\right)$ и подставим выражение для $t_{0}$ :

$$
\left\|P_{n}\right\|\left(120-20 \tau \frac{\left\|P_{n}\right\|-1}{\left\|P_{n}\right\|}+\tau^{2} \frac{\left(\left\|P_{n}\right\|-1\right)^{2}}{\left\|P_{n}\right\|^{2}}\right) \leq 120-20 \tau \frac{\left\|P_{n}\right\|-1}{n^{2}\left\|P_{n}\right\|} .
$$


Правая часть (2.15), очевидно, возрастает по $n$, поэтому можно подставить $n=3$, умножить на $\left\|P_{n}\right\|$ и сгруппировать относительно $\left\|P_{n}\right\|$ :

$$
\left\|P_{n}\right\|^{2}\left(120-20 \tau+\tau^{2}\right)+\left\|P_{n}\right\|\left(-120+\tau\left(20+\frac{20}{9}\right)-2 \tau^{2}\right)+\tau^{2}-\frac{20}{9} \tau \leq 0 .
$$

Нетрудно убедиться, что левая часть (2.16) возрастает относительно $\left\|P_{n}\right\|$. Следовательно, $\tau$ должно быть таким, чтобы неравенство (2.16) выполнялось для $\left\|P_{n}\right\|=1.5: \frac{1}{4} \tau^{2}-\frac{125}{9} \tau+90 \leq 0$. Нулями квадратного многочлена являются числа

$$
\tau_{1}=\frac{250-2 \sqrt{8335}}{9}<7.5, \quad \tau_{2}=\frac{250+2 \sqrt{8335}}{9}>48.07 .
$$

В итоге находим ограничения на $\tau: \tau_{1} \leq \tau \leq 18$.

Таким образом, получаем следующую оценку:

$$
\mu\left(\left\|P_{n}\right\| T_{n}^{\prime} / n^{2}\right) \leq 2\left(1-x_{0}\right)=2\left(1-\cos t_{0}\right)<2\left(1-1+t_{0}^{2} / 2\right)=t_{0}^{2}=\tau \frac{\left\|P_{n}\right\|-1}{\left\|P_{n}\right\| n^{2}} .
$$

Применяя (2.2) для меры множества, на котором многочлен превосходит 1, мы приходим к неравенству

$$
M_{n}\left(P_{n}\right)=\frac{\mu\left(P_{n}^{\prime} / n^{2}\right)}{\mu\left(P_{n}\right)} \leq \tau_{1}<7.5, \quad\left\|P_{n}\right\| \leq 1.5, n \geq 3 .
$$

Рассмотрим случай $n=2$. Уравнение $(2.7)$ принимает вид $4\left(2 x^{2}-1\right)+4 x=0$, его наибольший корень $\alpha_{2}=1 / 2$. Как следствие условие (2.10) сводится к неравенству $\left\|P_{2}\right\| \leq 2 \sqrt{1-1 / 4}=$ $\sqrt{3}$. Далее, если $\left\|P_{2}\right\| T_{2}^{\prime}\left(x_{0}\right)=\left\|P_{2}\right\| 4 x_{0}=2^{2}$, то $x_{0}=1 /\left\|P_{2}\right\|$. Отсюда $\mu\left(P_{2}^{\prime} / 4\right) \leq 2\left(1-x_{0}\right)=$ $2 \frac{\left\|P_{2}\right\|-1}{\left\|P_{2}\right\|}$, и в итоге

$$
M_{2}\left(P_{2}\right) \leq 2 \frac{\left\|P_{2}\right\|-1}{\left\|P_{2}\right\|} \frac{4\left\|P_{2}\right\|}{\left\|P_{2}\right\|-1}=8 .
$$

Таким образом, для всех $n \geq 2$ и $\left\|P_{n}\right\| \leq 1.5$ получаем $M_{n}\left(P_{n}\right) \leq 8$.

Докажем вторую оценку в (3). Как отмечено выше, если $x$ удовлетворяяет $(2.9)$, то $\left|P_{n}^{\prime}(x)\right|<$ $n^{2}$. Поэтому при условии $\left\|P_{n}\right\|<n$ справедлива оценка $\mu\left(P_{n}^{\prime} / n^{2}\right) \leq 2\left(1-\sqrt{1-\left\|P_{n}\right\|^{2} / n^{2}}\right)$, которая вкупе с (2.2) позволяет получить цепочку неравенств

$$
M_{n}\left(P_{n}\right)=\frac{\mu\left(P_{n}^{\prime} / n^{2}\right)}{\mu\left(P_{n}\right)} \leq \frac{2\left(1-\sqrt{1-\left\|P_{n}\right\|^{2} / n^{2}}\right)}{\left(\left\|P_{n}\right\|-1\right) /\left(n^{2}\left\|P_{n}\right\|\right)} \leq \frac{2\left\|P_{n}\right\|^{3}}{\left\|P_{n}\right\|-1} \leq \frac{2 c^{3}}{c-1} .
$$

Если $\left\|P_{n}\right\| \geq n$, то тривиальное замечание, что $\mu\left(P_{n}^{\prime} / n^{2}\right) \leq 2$, и (2.2) приводят к неравенству

$$
M_{n}\left(P_{n}\right)=\frac{\mu\left(P_{n}^{\prime} / n^{2}\right)}{\mu\left(P_{n}\right)} \leq \frac{2 n^{2}\left\|P_{n}\right\|}{\left\|P_{n}\right\|-1} \leq \frac{2 c^{3}}{c-1} .
$$

Теорема 2. Для $n \geq 2$ справедлива оченка $M_{n} \leq 6 n+1$.

Д о к а з а т е л ь с т в о. В виду теоремы 1 достаточно рассмотреть многочлены, у которых $\left\|P_{n}\right\|>1.5$. Определим два множества

$$
A=A\left(P_{n}\right):=\left\{x \in[-1,1]:\left|P_{n}(x)\right| \leq 1\right\}, \quad B=B\left(P_{n}\right):=\left\{x \in A:\left|P_{n}^{\prime}(x)\right|>n^{2}\right\} .
$$

Ясно, что $\mu\left(P_{n}^{\prime} / n^{2}\right)=M_{n}\left(P_{n}\right) \mu\left(P_{n}\right)$, поэтому mes $B\left(P_{n}\right) \geq \mu\left(P_{n}^{\prime} / n^{2}\right)-\mu\left(P_{n}\right) \geq\left(M_{n}(P)-\right.$ 1) $\mu\left(P_{n}\right)$. Далее, многочлен $P_{n}$ степени $n$ имеет не более $n$ промежутков монотонности, поэтому 
множество $A$ состоит не более чем из $n$ промежутков, интеграл от $\left|P_{n}^{\prime}\right|$ по каждому из которых не превосходит 2 , следовательно, $\int_{A}\left|P_{n}^{\prime}(x)\right| d x \leq 2 n$. Отсюда

$$
\begin{gathered}
2 n \geq \int_{A}\left|P_{n}^{\prime}(x)\right| d x \geq \int_{B}\left|P_{n}^{\prime}(x)\right| d x \geq n^{2} \operatorname{mes} B\left(P_{n}\right), \\
2 n \geq n^{2} \operatorname{mes} B\left(P_{n}\right) \geq n^{2}\left(M_{n}\left(P_{n}\right)-1\right) \mu\left(P_{n}\right) \geq n^{2}\left(M_{n}\left(P_{n}\right)-1\right) \frac{\left\|P_{n}\right\|-1}{n^{2}\left\|P_{n}\right\|} .
\end{gathered}
$$

Условие $\left\|P_{n}\right\|>1.5$ позволяет оценить $\left(\left\|P_{n}\right\|-1\right) /\left\|P_{n}\right\| \geq 1 / 3$. Тогда (2.17) принимает вид $2 n \geq\left(M_{n}\left(P_{n}\right)-1\right) / 3$, откуда $M_{n}\left(P_{n}\right) \leq 6 n+1$.

Теорема 3. Для $n \geq 2$ справедливо неравенство

$$
M_{n} \geq \lim _{b \rightarrow 1+} M_{n}\left(T_{n}(b x)\right)=1+\frac{3}{\left(n^{2}-1\right)} .
$$

Д о к а з а т е л ь с т в о. Рассмотрим многочлены вида $P_{n}(x)=T_{n}(b x)$, где $T_{n}(x)-$ многочлен Чебышева первого рода степени $n$ и $b>1$. В этом случае $\left|P_{n}(x)\right|=\left|T_{n}(b x)\right| \geq 1$ тогда и только тогда, когда $|b x| \geq 1$, т. е. $|x| \geq 1 / b$. Поэтому $\mu\left(P_{n}\right)=2(1-1 / b)$. Оценим $\mu\left(P_{n}^{\prime} / n^{2}\right)$. Заметим, что $P_{n}^{\prime}(1 / b)=b n^{2}, P_{n}^{\prime \prime}(1 / b)=b^{2} n^{2}\left(n^{2}-1\right) / 3$. Так как $P^{\prime \prime}(x)$ в малой окрестности $1 / b$ не убывает, то $P^{\prime \prime}(b x) \leq b^{2} n^{2}\left(n^{2}-1\right) / 3$ для любого $x \in[d, 1 / b]$, где $d-$ наибольшее из чисел, для которых $P_{n}^{\prime}(d)=n^{2}$. Положим $r:=1 / b-d$, тогда справедливо неравенство

$$
P_{n}^{\prime}(d)+P_{n}^{\prime \prime}(1 / b) r=n^{2}+b^{2} n^{2}(n-1)^{2} r / 3 \geq b n^{2}=P_{n}^{\prime}(1 / b) .
$$

Отсюда получаем

$$
r \geq \frac{3(b-1)}{b^{2}\left(n^{2}-1\right)}
$$

Производная превосходит $n^{2}$ по крайней мере на отрезке $[1 / b-r, 1]$ и симметричном ему $[-1, r-$ $1 / b, 1]$, следовательно, $\mu\left(P^{\prime} / n^{2}\right) \geq 2(1-1 / b+r)$. Таким образом, имеем оценки

$$
M_{n}\left(P_{n}\right) \geq \frac{(1-1 / b+r)}{(1-1 / b)} \geq 1+\frac{3}{b\left(n^{2}-1\right)}, \quad M_{n} \geq \lim _{b \rightarrow 1} \frac{3}{b\left(n^{2}-1\right)}+1=1+\frac{3}{\left(n^{2}-1\right)} .
$$

\section{СПИСОК ЛИТЕРАТУРЫ}

1. Sroka G. Constants in V.A. Markov's inequality in $L_{p}$ norms // J. Approx. Theory. 2015. Vol. 194. P. 27-34. doi: 10.1016/j.jat.2014.12.010.

2. Глазырина П.Ю. Точное неравенство Маркова - Никольского для алгебраических многочленов в пространствах $L_{q}, L_{0}$ на отрезке // Мат. заметки. 2008. Т. 84, № 1. С. 3-22.

3. Milovanovic G. V Mitrinovic D. S. Rassias Th. M. Topics in polynomials: extremal problems, inequalities, zeros. Singapore: World Sci. Publ., 1994. 821 p. doi: 10.1142/1284.

4. Goetgheluck P. On the Markov inequality in $L^{p}$-spaces // J. Approx. Theory. 1990. Vol. 62. P. 197-205. doi: 10.1016/0021-9045(90)90032-L .

5. Arestov V. V. Algebraic polynomials least deviating from zero in measure on a segment // Ukranian Math. J. Vol. 2010. Vol. 62, no. 3. P. 331-342. doi: 10.1007/s11253-010-0357-z .

6. Бабенко А. Г. Неравенства слабого типа для тригонометрических полиномов // Тр. Ин-та математики и механики УрО РАН. 1992. Т. 2 С. 34-41.

7. Лившиц Е. Д. Неравенство слабого типа для равномерно ограниченных тригонометрических полиномов // Тр. МИАН. 2013. Т. 280. C. 215-226. doi: 10.1134/S0371968513010147.

8. Вороновкая Е. В. Функционал первой производной и уточнение теоремы А. А. Маркова // Изв. АН СССР. Сер. математическая. 1959. Т. 23, № 6. С. 951-962. 
9. Гусев В. А. Функционалы производных от алгебраического полинома и теорема В. А. Маркова // Изв. АН СССР. Сер. математическая. 1961. Т 25, № 3. С. 367-384.

10. Вороновкая Е. В. Метод функционалов и его приложения. Ленинград: Изд-во Ленинградского электротехнического Ин-та связи, 1963. 181 с.

11. Borwein P., Erdeyi T. Polynomials and polynomial inequalities. 1995. 480 p. (Graduate Texts in Math.; vol. 161.) doi: 10.1137/1038150.

Поступила 2.04.2019

После доработки 13.05.2019

Принята к публикации 20.05.2019

Паюченко Никита Славич

младший науч. сотрудник

Институт математики и механики им. Н. Н. Красовского УрО РАН;

аспирант

Уральский федеральный университет

г. Екатеринбург

e-mail: aueiyo@gmail.com

\section{REFERENCES}

1. Sroka G. Constants in V.A. Markov's inequality in $L_{p}$ norms. J. Approx. Theory, 2015, vol. 194, pp. 27-34. doi: 10.1016/j.jat.2014.12.010.

2. Glazyrina P.Yu. The sharp Markov-Nikolskii inequality for algebraic polynomials in the spaces $L_{q}$ and $L_{0}$ on a closed interval. Math. Notes, 2008, vol. 84, no. 1, pp. 3-21. doi: 10.1134/S0001434608070018.

3. Milovanovic G.V Mitrinovic D.S. Rassias Th.M. Topics in Polynomials: extremal problems, inequalities, zeros. Singapore: World Sci. Publ., 1994, 821 p. doi: 10.1142/1284.

4. Goetgheluck P. On the Markov inequality in $L^{p}$-spaces. J. Approx. Theory, 1990, vol. 62, pp. 197-205. doi: 10.1016/0021-9045(90)90032-L .

5. Arestov V.V. Algebraic polynomials least deviating from zero in measure on a segment. Ukranian Math. J., 2010, vol. 62, no. 3, pp. 331-342. doi: 10.1007/s11253-010-0357-z .

6. Babenko A.G. Weak-type inequalities for trigonometric polynomials. Trudy Instituta Matematiki $i$ Mekhaniki URO RAN, 1992, vol. 2, pp. 34-41 (in Russian).

7. Livshits E.D. A weak-type inequality for uniformly bounded trigonometric polynomials. Proc. Steklov Inst. Math., 2013, vol. 280, no. 1, pp. 208-219. doi: 10.1134/S0371968513010147.

8. Voronovskaya E.V. The functional of the first derivative and improvement of a theorem of A.A. Markov. Izv. Akad. Nauk SSSR. Ser. Mat., 1959, vol. 23, no. 6, pp. 951-962 (in Russian).

9. Gusev V.A. Functionals of the derivatives of an algebraic polynomial and a theorem of V. A. Markov. Izv. Akad. Nauk SSSR Ser. Mat., 1961, vol. 25, no. 3, pp. 367-384 (in Russian).

10. Voronovskaya E.V. Metod funktsionalov $i$ ego prilozheniya [The method of functionals and its applications]. Leningrad: Leningradskii Elektrotekhnicheskii Institut Svyazi Publ., 1963, 181 p.

11. Borwein P., Erdeyi T. Polynomials and polynomial inequalities. Ser. Graduate Texts in Math., vol. 161, 1995, 480 p. doi: $10.1137 / 1038150$.

Received April 2, 2019

Revised May 13, 2019

Accepted May 20, 2019

Funding Agency: This work was supported by the Russian Academic Excellence Project (agreement no. 02.A03.21.0006 of August 27, 2013, between the Ministry of Education and Science of the Russian Federation and Ural Federal University).

Nikita Slavich Pauchenko, Krasovskii Institute of Mathematics and Mechanics of the Ural Branch of the Russian Academy of Sciences, Yekaterinburg, 620108 Russia; Ural Federal University, Yekaterinburg, 620002 Russia, e-mail: aueiyo@gmail.com .

Cite this article as: N. S. Pauchenko. Markov's weak inequality for algebraic polynomials on a closed interval, Trudy Instituta Matematiki i Mekhaniki URO RAN, 2019, vol. 25, no. 2, pp. 160-166. 